\title{
Beiträge zu dem Problem des Geburtenüberschusses der Knaben.
}

\author{
Von
}

\author{
Dr. med. A. Gottstein \\ in Berlin.
}

Bei Gelegenheit meiner Untersuchungen über den Einfluss der Epidemien auf die Kindersterblichkeit musste ich auch die bekannte Thatsache des ungleichen Betroffenseins beider Geschlechter und die verschiedenen Versuche zur Erklärung dieser an sich durchaus nicht selbstverständlichen Erscheinung zum Gegenstande meines Studiums machen. Bei weiterer Vertiefung erwuchs dann für mich die Nothwendigkeit, einem der ältesten Probleme der Naturwissenschaft näher zu treten, nämlich der Frage nach den Ursachen des gesetzmässigen Ueberschusses der männlichen Geburten gegenüber den weiblichen beim menschlichen Geschlechte.

Die Thatsache selbst, dass unter den Geburten die Zahl der männlichen Kinder in einem ganz bestimmten Verhältniss überwiegt, gehört zu den sichersten Errungenschaften der Wissenschaft. Ueberhaupt scheint bei jeder Thiergattung das Verhältnis zwischen den Geburtenzahlen beider Geschlechter eine constante Grösse zu sein. Dies führte schon Hofacker aus, welcher ungefähr gleichzeitig mit Sadler eine durch statistische Belege gestützte Hypothese zur Erklärung dieser Erscheinung aufstellte. Es finden sich nämlich nach Hofacker ${ }^{l}$ bei den verschiedenen Thiergattungen die allerverschiedensten Verhältnisse der Betheiligung beider Geschlechter an der Geburtenzahl von der annähernden Gleichheit bis zum schwachen oder ganz erheblichen Ueberwiegen des einen oder des anderen Geschlechtes. Beim menschlichen Geschlechte beträgt gegenwärtig in Europa, soweit zuverlässiges statistisches Material vorliegt, die ,Sexualproportion“" auf

${ }^{1} \mathrm{H}$ ofacker. Deber die Eigenschaften, welche sich bei Menschen und Thieren von den Eltern auf die Nachkommen vererben. Tübingen, Osiander, 1828. S. 43. 
100 Mädchengeburten 106.3 Knabengeburten. Man kann dies Verhältniss auch mit J. Lehr ${ }^{1}$ dadurch ausdrücken, dass man die Zahl der Mädchengeburten durch die aller Geburten dividirt; dann beträgt der Antheil der Mädchengeburten 0,48508 . Leh r weist durch Formeln der Wahrscheinlichkeitsrechnung nach, dass die an seinem Materiale beobachteten $A b-$ weichungen von dieser Zahl, die zu verschiedenen Zeiten und an verschiedenen Orten festgestellt wurden, dem Gesetze des Zufalls unterworfen sind und in den Bereich der natürlichen Fehler fallen. Gegen die Beweisführung von Lehr hat allerdings v. Fircks ${ }^{2}$ Bedenken erhoben; diese beziehen sich aber nur auf die Brauchbarkeit des von Lehr benutzten statistischen Materiales, richten sich jedoch selbstverständlich nicht gegen das Gesetz von der Sexualproportion.

Die Regeln der Wahrscheinlichkeitsrechnung lassen erwarten, dass, wenn für zwei Vorgänge die Möglichkeit ihres Eintretens von den gleichen Bedingungen abhängt, also gleich gross ist, bei einer sehr grossen Zahl von Fällen beide Ereignisse gleichmässig häufig eintreten müssen. Wenn in einer verdeckten Urne je eine weisse und eine schwarze Kugel liegen und beliebig oft jedesmal eine Kugel der Urne entnommen wird, so muss z. B. bei 10000 Ziehungen ebenso oft eine schwarze, wie eine weisse Kugel gezogen werden. Wenn nach Abschluss dieses Versuchs dagegen eine Ungleichheit sich ergiebt, welche die Fehlergrenzen überschreitet, wenn entweder die schwarze oder die weisse Kugel häufiger gezogen werden, so ist die nothwendige Folgerung die, dass ausser dem Zufall noch andere ursächliche Momente an dem Ergebniss bestimmend mitgewirkt haben müssen. Wenn also für das Eintreten einer Knaben- oder einer Mädchengeburt die Wahrscheinlichkeit beider Ereignisse gleich gross ist, so muss erwartet werden, dass an einem grossen Materiale die Zahl beider Geschlechter gleich gross wird. Ergiebt die Beobachtung jedoch eine Ungleichheit, wie sie thatsächlich mit der Regelmässigkeit eines Naturgesetzes auftritt, so entsteht die nothwendige Folgerung, dass ausser dem Zufall nogh andere uns unbekannte Ursachen mitwirken müssen, welche diese gesetzmässige Ungleichheit hervorrufen. Es musste daher diese, seit dem Bestehen sorgfältiger Beobachtung gleichmässig festgestellte Erscheinung die grösste Beachtung der Forscher finden. Denn die Reihenfolge von Knabengeburten oder Mädchengeburten war zwar im Einzelfalle, wie er sich aus der Betrachtung einer geringen Anzahl von Ehen ergab, scheinbar durchaus der Willkür unterworfen; bei der Betrachtung grosser Zahlen aber ergab sich eine Gesetzmässigkeit, welche gerade bei diesem Gegen-

\footnotetext{
1 J. Lehr. Zeitschrift für die gesammten Staatswissenschaften. 1889.

${ }^{2}$ v. Fircks. Zeitschrift des preussischen statistischen Amtes. 1889.
} 
stande viele Bearbeiter der Frage immer wieder das Wort von Süssmilch citiren lässt, - „, von der Ordnung, die sich durch die Unordnungen im Kleinen verbirgt". Wenn man übrigens an einem grossen Materiale das Problem unter Berücksichtigung besonderer Beziehungen einer noch weiteren Theilung unterwirft, so lassen sich, wie Geissle $r^{1}$ nachwies, sogar noch speciellere gesetzmässige Erscheinungen nachweisen. Zunächst aber besehränkt sich unser Interesse auf die allgemeine Thatsache des gesetzmässigen Eintretens dieser Sexualproportion, bei der es sich um ein biologisches Gesetz ersten Ranges handelt. Dies exact zu beweisen, hielt kein Geringerer als Laplace ${ }^{2}$ für einen würdigen Gegenstand seiner mathematischen Betrachtungen. In seinem klassischen Werke von der Wahrscheinlichkeit weist Laplace nach, dass der Ueberschuss der Knabengeburten eine Erscheinung sei, von welcher durch die Wahrseheinlichkeitsrechnung dargethan werden könne, dass sie „extrêmement approchante de la certitude" sei. Interesssant ist, dass er bei der Betrachtung der europäischen Grossstädte die Sexualproportion in Paris zwar auch zu Gunsten der Knabengeburten, aber in geringerer Höhe als in London oder Neapel findet. Schon diese geringe Differenz von 104 gegenüber der soust beobachteten Zahl von 106 erscheint einem Laplace nicht durch den Zufall bedingt, sondern durch besondere Ursachen veranlasst, und er ruht nicht eher, als bis er den Grund dieser Abweichung entdeckt hat. Er findet ihn in dem zahlenmässig nachweisbaren Missbrauch der Landbevölkerung um Paris, ihre Kuabengeburten zwar selbst aufzuerziehen, die weniger werthvollen Mädchen aber einfach als Findlinge in Paris auszusetzen, wo sie die Zahl der Mädehengeburten scheinbar vermehren.

Freilich haben bisher die zahlreichen Versuche, die Ursache der Sexualproportion aufzudecken, noch zu keiner befriedigenden Lösung geführt. Ja, die Mehrzahl dieser Erklärungsversuche ist geradezu unwissenschaftlich und phantastisch, um so mehr dann, wenn sie mit der Tendenz verquickt sind, im Einzelfalle den Geschlechtscharakter des neu erzeugten Wesens auf ein bestimmtes Verhalten des Erzeugers zurückführen zu wollen. Für alle diese Spielereien, deren einige in jüngster Zeit wieder aufgetaucht sind, gilt das Wort von Geissler, ${ }^{3}$ dass ,in dem vorhandenen Ueberschusse der Knabengeburten ein weit mächtigeres Naturgesetz verborgen ist, als es z. B. die Anhänger jener Meinung annehmen möchten, welche hier nur die Wirkung des Wunsches, männliche Nachkommen zu erzeugen, ausgedrückt finden".

${ }^{1}$ Geissler, Beiträge zur Frage des Geschlechtsverhältnisses der Geborenen. Zeitschrift des königl. sächs. statistischen Bureaus. 1889.

'Laplace. Théorie analythique des probabilités. Paris, Courcier, 1812. S. $377 \mathrm{ff}$.

3 A. a. 0 . 
Am meisten Beachtung hat noch die schon genannte HofackerSadler'sche Hypothese gefunden, die auf thatsächliches Material und nüchterne Erwägungen sich stützt. Hofacker begründet seine Theorie durch die Beobachtungen an Lämmerherden, Gestüten und an einem verhältnissmässig geringen Material menschlicher Ehen. Auf dieșe Daten gestützt, baut er die Theorie auf, dass der Altersuntersehied der Erzeuger wesentlich bestimmend für das Geschlecht sei, wobei andere Momente, wie z. B. die Stärke der Constitution des Erzeugenden, durchaus nicht ausgeschlossen würden. ${ }^{1}$ Und zwar überwiegt nach ihm in der Geburtenfolge das Geschlecht des älteren Erzeugers; da nun beim Menschen der Ehemann gewöhnlich älter sei, so erkläre sich hieraus bei Betrachtung eines grösseren Materiales der Geburtenüberschuss der Knaben.

Von mehr theoretischen Gesichtspunkten ausgehend, kam Sadler ${ }^{2}$ in seinem grossen Werke über das Bevölkerungsgesetz unabhängig von Hofacker zur Aufstellung einer nahezu gleichen Hypothese. Er geht von der Weisheit der Natur aus, welche unmöglich gewollt haben könne, dass, wenn das Verhältniss der Knabengeburten zu dem der Mädchengeburten dasjenige von 25:24 sei, ein Fünfundzwanzigstel der Männer zur Ehelosigkeit verurtheilt würde. Denn es sei der Zweck der Natur, zur Erzeugung neuer menschlicher Wesen die gleiche Zahl männlicher und weiblicher Individuen in erzeugungsfähigem Alter zusammenzubringen. Nun sei aber sowohl die Gefährdung der Geschlechter bis zur Erreichung dieses Alters eine ungleiche, als auch der Eintritt und die Dauer der Generationsperiode durchaus verschieden. Die Sterblichkeit der Knaben sei eine grössere, und zwar zeige sich diese Erscheinung von den Todtgeburten bis zur Erreichung der Zeugungsf̣ähigkeit. Ausserdem träte die Zeugungsfähigkeit der Männer später ein und dauerte länger; gerade dieser Umstand aber sei deshalb wichtig, weil nicht nur einmalige Verehelichungen, sondern zwei- und mehrfache vorkämen; hier sei es aber statistisch nachweisbar, dass mehr Wittwer als Wittwen sich wieder verheiratheten. Um also den Naturzweck zu erreichen, dass der gleichen Zahl Frauen in vermehrungsfähigem Alter die gleiche Anzahl Männer gegenüberstände, sei wegen des grösseren Verlustes an Knaben ein Ueberschuss der Knabengeburten erforderlich. In dieser ganzen, rein teleologischen Deduction ist nur der eine Gedanke ausserordentlich glücklich, die Geburtenzahl der Geschlechter nicht absolut zu betrachten, sondern relativ in ihrer Beziehung zum späteren, fortpflanzungsfähigen Alter.

A. a. O. S. 57.

Sadler. The Law of population. London, Murray, 1830. Bd. Ir. Buch III. S. $317 \mathrm{ff}$. 
Dieser Gedanke ist aber sehr naheliegend, und es ist daher nicht wunderbar, dass Sadler für ihn nicht die Priorität besitzt. Auch Süssmilch hatte der gleichen Anschauung schon früher Ausdruck gegeben; auch er sieht in der grösseren Knabensterblichkeit vor Erreichung des zeugungsfähigen Alters teleologisch den Grund für den Ueberschuss der Knabengeburten. Die von Sadler angeführten Gründe sind aber, wie später angeführt werden soll, nicht einmal ganz richtig, und zudem macht er mit seinen Folgerungen zu früh Halt, während erst die Fortsetzung der Deduction auf dem Boden seines glücklichen Grundgedankens zu brauchbaren Ergebnissen geführt hätte.

Auf solche Erwägungen gestützt, ging nun Sadler zur Prüfung des vorhandenen Zahlenmaterials und stellte an der Hand der englischen Statistik unter Heranziehung der Verhältnisse verschiedenartiger Bevölkerungsschichten folgenden Satz auf: „Das Verhältniss, in welchem die Geschlechter geboren werden, wird geleitet und regulirt durch die Differenz im Alter ihrer Erzeuger, derart, dass durchschnittlich unter der Gesammtzahl der Geburten das Geschlecht des älteren Erzeugers überwiegt; der Ueberschuss des einen Geschlechts geht ferner mit dem Unterschied in der Sterblichkeit beider Geschlechter parallel, so dass bei der Erreichung des heirathsfähigen, unter. sich verschiedenen Alters beider Geschlechter deren Zahl balancirt." Neben anderem Beweismaterial schien eine Hauptstütze der Sadler'schen Theorie durch die vor ihm betonte auffällige Thatsachė gegeben, dass bei den unehelichen Geburten, bei denen, anders als bei den ehelichen, die Altersunterschiede zu Ungunsten des Mannes häufig geringer sind, die Zahl der Kinder weiblichen Geschlechts grösser sein sollte, als bei den ehelichen Geburten, und dass hier sogar nahezu Gleichheit des Geburtenverhältnisses bestände.

Trotzdem sich viele Statistiker späterer Jahrzehnte für diese Theorie erklärten, hat sie sich bei Massenbeobachtung nicht durchaus bestätigt. Jedoch, selbst wenn sie durchweg zu Recht bestände, wäre sie nichts weiter, als eine Umsehreibung von Thatsachen, nicht aber eine kausale Aufklärung einer auffallenden, gesetzmässigen Erscheinung.

Bei meinen eigenen Studien über das vorliegende Problem bin ich auf Beziehungen gestossen, welche dasselbe dem Verständniss ausserordentlich viel näher bringen. Diese Beziehungen sind so elementare und naheliegende, dass es mir in höchstem Grade wahrscheinlich ist, es müssten dieselben bei dem Umfang der Forschungen über diesen Gegenstand schon wiederholt von anderer Seite aufgedeckt worden sein. Da ich jedoch bei der Einsicht in die vorhandene Litteratur nirgends auch nur eine Andentung oder einen Hinweis auf diese Beziehungen gefunden habe, so muss ich annehmen, dass die von mir gefundene Lösung wenigstens in 
weiteren Kreisen nicht bekannt oder wieder verloren gegangen ist, und dass eine Darstellung derselben, selbst wenn sie nur eine Wiederauffindung bedeuten sollte, am Platze ist.

Wie bei so vielen schwer oder gar nicht lösbar erscheinenden Problemen liegt auch hier das Hinderniss nur an einer nicht ganz richtigen Fragestellung und fällt bei Beseitigung dieses Fehlers sofort hinweg.

Um das Princip der Lösung schon hier vorweg zu nehmen, so soll zunächst von allen complicirenden oder gar von teleologischen Momenten, welche frühere Erklärer betont haben, wie vor Allem von der grösseren Knabensterblichkeit, durchaus abgesehen werden. Wenn wir uns an die Forderungen der Wahrscheinlichkeitsgesetze halten, so verlangen diese bei Zusammenfassung eines grösseren Materials eine absolute Gleichheit der männlichen und weiblichen lebenden Individuen; besteht diese Gleichheit nicht, so muss man schliessen, dass bei deren Erzeugung besondere Ursachen bestimmend mitgewirkt haben, welche das Ueberwiegen des einen oder des anderen Geschlechts bedingten. Umgekehrt aber schliesst der Nachweis der Gleichheit in dem Verhältniss der beiden Geschlechter das Bestehen solcher Ursachen ohne Weiteres aus. Die principielle Frage ist nun aber die, welche Maasseinheit für die Bestimmung des Vorhandenseins einer gleichen Zahl männlicher und weiblicher Individuen der Betrachtung zu Grunde gelegt werden muss. Das bisher gebräuchliche Verfahren ist dasjenige, dass an einem grösseren Materiale die Zabl der in der Zeiteinheit stattfindenden Geburten beider Geschlechter einander gegenüber gestellt werden, nicht aber die Zahl der Lebenden aller Altersclassen, an welcher mindestens drei aufeinander folgende Generationen betheiligt sind, neben sämmtlichen überhaupt möglichen Zwischenstufen dieser drei Generationen. Als Zeiteinheit dient für gewöhnlich das bürgerliche Jahr oder ein Theil oder ein Vielfaches dieses Maasses. Eine jede Geburt stellt nun das Product des Zusammenwirkens je eines Vertreters beider Geschlechter und ferner den Anfang einer neuen Generation vor. Eine Summe von Geburten, welche in der Zeiteinheit gleichzeitig eintreten, geht also auf das gleichzeitige Vorhandensein der doppelten Zahl geschlechtsreifer Individuen zurück und in dieser Zahl sind beide Geschlechter zu gleichen Theilen vertreten. Die neugeborenen Individuen wachsen aber wieder zu geschlechtsreifen fortpflanzungsfähigen Wesen heran; sie erreichen jedoch je nach ihrem Geschlechte diese Zeit zu ganz verschiedenen Zeitabschnitten, von ibrer Geburt an gerechnet, nämlich die Mädchen früher, die Knaben später. Auch die Eltern, aus deren Ehen die gerade in der Zeiteinheit der Betrachtung unterzogenen Individuen 
hervorgegangen sind und welche im Augenblicke der Untersuchung gleichzeitig geschlechtsreif gewesen sind, haben ihrem Geschlechte entsprechend von ihrer Geburt bis zur Erzeugung eines Nachwuchses einen verschieden grossen Zeitraum durchlaufen. Die bisherige Untersuchung der Frage von der Sexualproportion geht also von der Betrachtung des Verhältnisses je zweier auf einander folgender Generationen aus, von denen die erste den Weg von der Geburt bis zur Geschlechtsreife schon zurückgelegt hat, die zweite dagegen eben zu beginnen sich anschickt. Dieser Weg ist aber für die Vertreter eines jeden Geschlechtes verschieden gross, für die männlichen Geburten länger als für die weiblichen. Die Untersuchung der Frage, ob die Zahl der männlichen und der weiblichen Existenzen gleich gross ist oder nicht, geht also in der jetzt üblich gewordenen Form der Prüfung schon von einer Ungleichheit der Verhältnisse beider Gesehlechter in der Voraussetzung aus, welche, wenn man sich auf die Betrachtung nur zweier unmittelbar auf einander folgender Generationen in verschiedenen Stadien ihrer Entwickelung beschränkt, keine Berücksichtigung findet und daher $\mathrm{zu}$ schweren Urtheilstäuschungen führen muss. Diese schon in der Voraussetzung enthaltene Ungleichheit ist eben die verschiedene Generationsdauer der beiden Geschlechter, eine für die Erwägung so nahe liegende Thatsache, dass schon Süssmilch und Sadler ihrer gedacht haben. Trotzdem haben beide Forseher die Folgerungen nicht gezogen, die sich nothwendig ergeben, wenn man die Einwirkung der ungleichen Generationsdauer beider Geschlechter nicht nur bei zwei auf einander folgenden Generationen, also bei der Betrachtung des Verhältnisses zwischen Eltern und Kind, sondern in der Aufeinanderfolge zahlreicher Geschlechter weiter verfolgt.

Vor der Wiedergabe des exacten Beweises sei die Anführung eines einfachen Beispieles zur Erleichterung des Verständnisses gestattet. Angenommen, ein Ehepaar, welches im gewöhnlichen Lebensalter, der Mann mit 30, die Frau mit 20 Jahren geheirathet hat, erzeugte in den ersten 2 Jahren ihrer Ehe die gleiche Anzahl Vertreter beider Geschlechter, und zwar der Einfachheit halber je einen Sohn und eine Tochter. Diese Kinder heiratheten mit Erreichung des ihrem Geschlecht entsprechenden Lebensalters und pflanzten sich in gleicher Weise zur Erzeugung der dritten Generation fort; diese dritte und auch die vierte Generation entwickelte. sich in gleicher Weise weiter, so dass in jeder neuen Generation die beiden Geschlechter an dem Nachwuchs gleichmässig betheiligt wären. Damn wird jenem ersten Elternpaare bei der ungleichen Zeitdauer, welche der männliche und der weibliche Nachwuchs bis zur Erreichung der Fortpflanzungsreife beanspruchen, auf der ausschliesslichen weiblichen Fortpflanzungslinie ungefähr zu dem gleichen Zeitpunkte die erste Ur- 
enkelin geboren werden, zu welchem auf der ausschliesslichen Sohneslinie erst der erste männliche Enkel geboren wird.

Für die mathematische Erklärung des Satzes liegen die Verbältnisse sehr complicirt. Zur Erzengung je eines neuen Lebewesens der auf einander folgenden Generationen ist immer die Zusammenfügung je eines Vertreters beider Geschlechter erforderlich. Ausserdem gehen aus einer Ehe nicht nur zwei, sondern mehr als zwei Nachkömmlinge durchschnittlich hervor. Es empfiehlt sich daher, die principielle Seite der Frage erst an

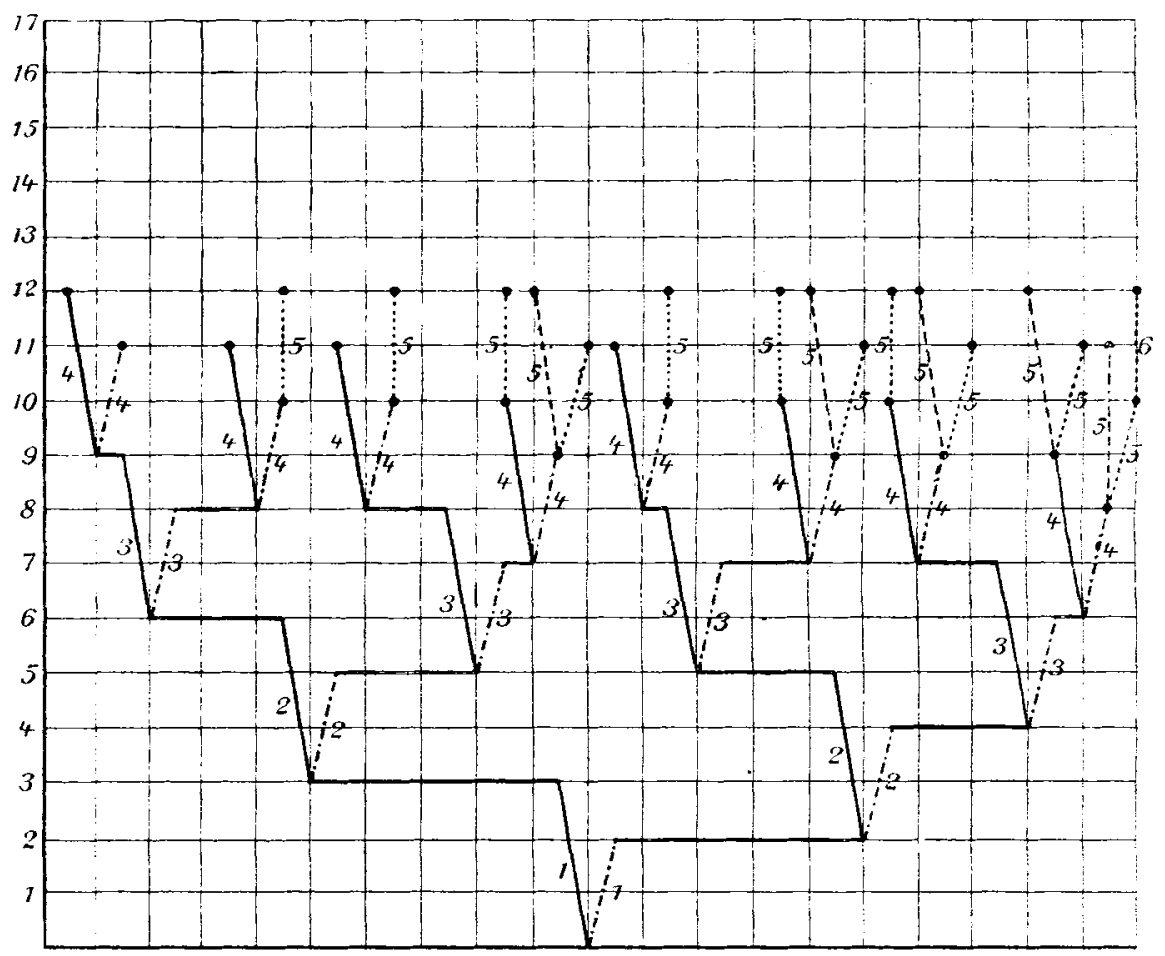

Fig. 1.

einem einfachen Beispiele zu entwickeln, bei welchem das Zusammenwirken zweier Individuen noch ausser Betracht bleibt. Angenommen der Stamm eines Baumes theile sich unbegrenzt dichotomisch in je zwei Aeste; diese zwei Aeste aber seien von ungleicher Länge und zwar sei bei jeder neuen Theilung das Verhältniss zwischen den beiden Aesten $a$ und $b$, von denen $a$ der kürzere sei, ein constantes. Das Wachsthum der beiden Theile sei das gleich schnelle, so dass der längere Ast immer in einem bestimmten Verhältniss später zur Theilung gelange, als der kürzere Ast. In der schematischen Zeichnung (Fig. 1) ist diese Theilung auf ein 
Coordinatensystem bezogen, bei welchem die Abschnitte der Ordinate die Zeitabschnitte bedeuten, zu welchen die Theilungen erfolgen. Nach einer bestimmten Zahl von Theilungen stellen sich zwei Extreme heraus, nämlich eine Reihe von Theilungen, welche nur aus den kurzen Aesten $a$ und eine solche, welche nur aus den langen Aesten $b$ bestehen. Die Theilungen über die ausschliesslich kurzen Aeste bestehen aus mehr Gliedern bis zur Erreichung der gewählten Höhengrenze, als diejenigen über die ausschliessliche $b$-Linie, und zwar verhalten sich diese Theilungen von $a$ und $b$ wie $b: a$.

In der schematischen Zeichnung der Fig. 1 verhalten sich die kurzen Aeste $a$ zu den langen Aesten $b$ wie 2:3. Bei dem Punkte 12 der Ordinate, welcher ein Vielfaches von $a$ und $b$ ausdrückt, haben die nur aus langen Aesten bestehenden Theilungen erst die vierte Generation erreicht, während die nur aus kurzen Aesten bestehenden Theilungen schon bei der sechsten Generation angelangt sind.

Nun folgt aber nicht stets auf einen langen Ast wieder ein langer und auf einen kurzen wieder ein kurzer; es bestehen nicht bloss die beiden Extreme, die entweder nur aus langen oder nur aus kurzen Aesten zusammengesetzt sind. Vielmehr werden auch gleichzeitig alle dazwischen liegenden Combinationen gebildet; neben dem Theilungsaste, der bei $n$ T'heilungen aus $n$ kurzen Aesten besteht, findet sich zunächst noch ein solcher, der aus $n-1$ kurzen und aus einem langen Aste besteht. Dieser eine lange Ast kann aber an den verschiedensten Stellen von der ersten bis zur letzten der $n$ Theilungen zu stehen kommen; der Ast von der Länge $(n-1) a 1 b$ wird sich also in $n$ facher Zahl vorfinden.

Wenn man nun die Theilung der Aeste des Baumes vollständig bis zu Ende führt, so sind gleich viel Aeste von a Länge und von $b$ Länge vorhanden. Aber sie vertheilen sich verschieden. In der unteren, dem Stamm nahe liegenden Hälfte überwiegen die kürzeren, in der oberen dagegen die längeren Aeste. Wenn man aber, wie in der Fig. 1, die Theilungen nicht bis in's Unendliche fortgehend sich denkt, sondern an einem beliebigen Punkte der Theilungen, also nach $n$ Theilungen, wobei für $n$ jeder Werth beigesetzt werden kann, eine Parallele zur Abscissenaxe zieht, so sind in dem zwischen ihr und der Abscisse eingeschlossenen Raum mehr Aeste von der Länge $a$, als von der Länge $b$ enthalten. In dem gewählten Beispiele des Baumes, der sich in's Unendliche fortgesetzt in je zwei Aeste von ungleicher länge theilt, von denen der längere Ast später zur Theilung gelangt als der kürzere, muss also, trotzdem alle gleichzeitig sich theilenden Aeste je einen kurzen und einen langen Ast 
abgeben, dennoch nach wenigen Generationen eine Ungleichheit des vorhandenen Aeste zu Gunsten des Ueberwiegens der kürzeren eintreten.

Die mathematische Lösung der Frage nach dem Verhältniss, in welchem nach $n$ Theilungen der einen Art die Zahl der kurzen zu der der langen Aeste steht, überschreitet die elementaren Methoden. Es ist hier nicht der Ort, complicirte mathematische Probleme zu lösen; immerhin ist das Problem einer mathematischen Behandlung zugänglich. Aber man kann sich an der Hand des Schemas der Figur 1 auch rein empirisch durch Zählung davon überzeugen, dass die Voraussetzung der Theilung in je zwei Aeste ungleicher Länge nach einer beliebigen Anzahl von Theilungsgenerationen eine Ungleichheit in 'dem Verhältniss der Gesammtzahl herbeiführt, in welchem die kürzeren Aeste an Zahl überwiegen müssen. Und zwar hängt das Verhältniss, in welchem die beiden ungleich langen Aeste sich bilden, von dem Verhältniss ihrer Länge ab. Umgekehrt kann eine Gleichheit in der Zahl der beiden ungleich langen Aeste bei Zusammenfassung mehrerer Theilungsgenerationen nur unter einer Bedingung erreicht werden; es muss bei jeder Theilung eine ungleiche Zahl langer und kurzer Aeste abgegeben werden und zwar müssen mehr lange Aeste bei jeder Theilung entstehen, als kurze. Und auch hier hängt wieder das Verhältniss der Zahl der gebildeten Aeste beider Arten von dem Verhältniss ihrer Länge ab.

Das Beispiel des sich theilenden Baumes lässt sich nicht ohne Weiteres auf die Fortpflanzungsart des menschlichen Geschlechtes übertragen. Denn während hier schon je ein Ast ausreicht, um sich zu theilen, müssen sich bei der Fortpflanzung der menschlichen Rasse je zwei Individuen verschiedenen Geschlechts und verschiedenen Alters zusammenfinden. Ausserdem ist deren Vermehrung nicht beendet, sobald sie zwei Aeste ungleicher Länge abgegeben haben. Denn aus der Ehe entspringen durchschnittlich mehr als je zwei Individuen. Trotzdem ist auch hier die Aufgabe nicht unlösbar, wenn deren Voraussetzung die Annahme ist, dass die Vertreter beider Geschlechter gesetzmässig in einem durchschnittlich ungleichen Lebensalter zur Fortpflanzung gelangen, denn die Aufgabe lässt sich ohne Weiteres auf den ersten Fall zurückführen.

In der zweiten Zeichnung (Fig. 2) bedeutet der Punkt $A$ ein Ehepaar, und es sei vorläufig vorausgesetzt, dass aus einer jeden Ehe in kurzer, der Zeit nach zu vernachlässigender Aufeinauderfolge je ein Knabe und ein Mädehen geboren würde, dass also in einer Vielheit von Ehen die Zahl der Mädchengaburten und der Knabengeburten in der Zeiteinheit gleich gross sei. Das Verbältniss der Generationsdauer sei wieder als constant vorausgesetzt und gleich den Ordinatenabschnitten 2 und 3 , welche Zeitabschnitte bedeuten. Dieses Ehepaar besitzt nun je ein Eltern- 
paar, aus Vater und Mutter bestehend, die wieder in dem Verhältniss von $a$ und $b$ verschiedene Zeiträume von der Geburt bis zur Verehelichung zu durchlaufen haben. Die Eltern der Frau haben demnach vor a Jahren, diejenigen des Mannes erst vor $b$ Jahren eben den Nachwuchs erzeugt, der jetzt zur Ehe sich zusammengefunden. Die gleichen Bedin-

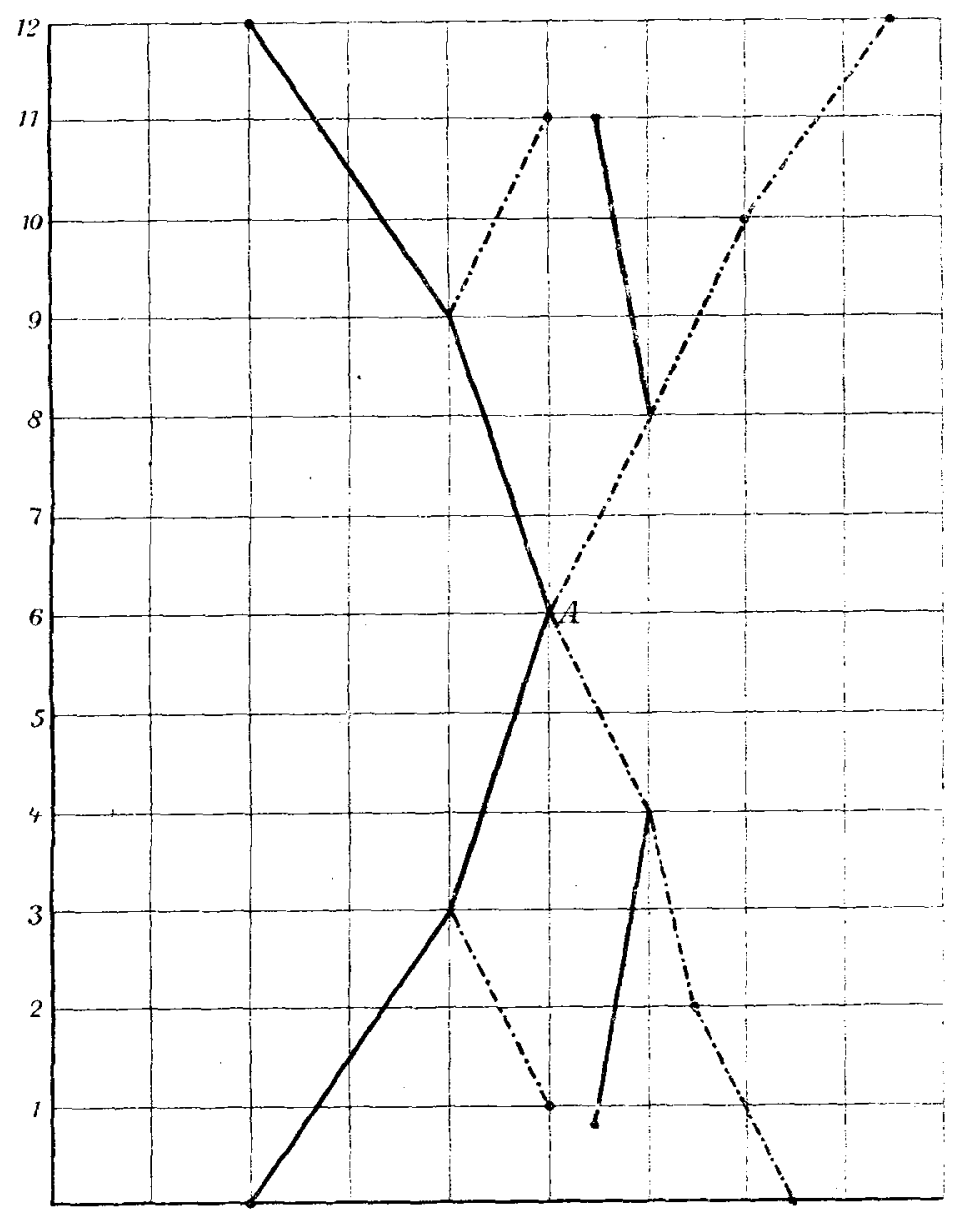

Fig. 2.

gungen gelten aber auch für diese zwei Elternpaare und wenn man die Zeichnung fortsetzt, so erhält man das gleiche Bild, wie in Fig. 1, nur dass jetzt die Zeitabschnitte zurück auf die Vergangenheit berechnet sind, während sie im ersten Fall vorwärts galten. Jedes dieser Elternpaare aber hat nach Voraussetzung nicht nur denjenigen Sprossen erzeugt, der 
in der Zeichnung nach der Abscissenaxe zu schon aufgeführt wurde, sondern auch einen zweiten Sprossen anderen Geschlechtes, der wieder den gleichen Bedingungen unterworfen ist. Von einem jeden Punkte der schematischen Zeichnung, der ein Ehepaar bedeutet, gehen also je zwei Linien von der Länge $a$ und $b$ nach oben und nach unten. Wenn man nun eine beliebige Fläche des Coordinatensystems durch Parallelen zu dem Axensystem abgrenzt, so bedeutet diese Fläche einen Zeitabschnitt, der zahlreiche auf einander und durch einander folgende Generationen von Ehepaaren einschliesst. Man kann sich diesen Zeitabschnitt von unendlich vielen Punkten ausgefüllt denken, deren jeder ein Ehepaar im Zeitpunkt der Geburt eines neuen Individuums bedeutet. Eine Parallele zur Abscisse umfasst diejenigen Ehepaare, welche in der Zeiteinheit gleichzeitig ein Kind produciren. Wenn nun für diese Geburten das Gesetz gültig ist, dass die Zahl der Knabengeburten gleich der der Mädchengeburten sei, so würde die Zeichnung zu Recht bestehen, dass von jedem Punkte je eine $a$-Linie und eine $b$-Linie nach oben wie nach unten ginge.

Von jedem beliebigen Punkte der durch das Coordinatensystem abgegrenzten Fläche kann man sich also sowohl senkrecht auf die Abscisse $\mathrm{zu}$, als in entgegengesetzter Richtung, je eine Figur von der Beschaffenheit der Fig. 1 ausgeführt denken. Jede dieser Verzweigungen gehorcht dem Gesetze, dass die Zahl der kürzeren Aeste in einem durch eine Parallele zur Abscisse begrenzten Raum überwiegt. Jeder dieser Punkte bedeutet den Zeitpunkt des Eintretens einer Geburt ohne Rücksicht auf die Eltern, aus deren Vereinigung sie sich herleitete. Da die Zahl der Punkte unendlich gross und die Wahl derselben beliebig ist, so fallen in diesem Schema alle complicirenden Momente aus, vor Allem der Umstand, dass die durchschnittliche Geburtszahl einer Ehe die Zahl zwei übersteigt. Streng durchgeführt ist dagegen die für den indirecten Beweis erforderliche Voraussetzung von dem gleichen Antheil der Geschlechter an der Geburtenzahl in der Zeiteinheit. Da aber die Folge dieser Voraussetzung nur die Bildung unendlich vielfacher Verzweigungen vom Schema der Fig. 1 ist, so muss eben die gleiche Betheiligung der Geschlechter bei den Geburten in der Zeiteinheit zu einem Ueberwiegen des Geschlechtes mit der kürzeren Generationsdauer in der Gesammtzahl der Lebenden in der Zeiteinheit führen.

Mit dieser Ausführung ist das Problem der "Sexualproportion" schon gelöst. Teleologische Betrachtungen, wie diejenige des Zwecks der Natur, der für jeden geschlechtsreifen Mann ein geschlechtsreifes Weib zu liefern beabsichtige, oder der gar bewusst die grössere Sterblichkeit der Knaben ausgleichen und die weitergehende Neigung der Wittwer zur Wiederverehelichung berücksichtigen wollte, haben hier keinen Platz. Die Ge- 
setze der Wahrscheinlichkeitsrechnung verlangen einfach, wenn kein zureichender Grund für das Vorhandensein einer grösseren Zahl der Glieder des einen oder des anderen Geschlechtes vorliegt, die absolute Gleichheit in der Zahl ihrer lebenden Vertreter. $\mathrm{Ob}$ eine solche besteht, dafür ist bisher irrthümlich eine falsche Prüfungsmethode angewendet worden, nämlich die Vergleichung der Antheile beider Geschlechter an der Geburtenzahl in der Zeiteinheit. Dieses Maass aber berücksichtigt eine schon in der Voraussetzung liegende Ungleichheit nicht, nämlich die ungleiche Generationsdauer beider Geschlechter, welche eine schnellere Regeneration des weiblichen Geschlechtes hervorrufen muss. Wäre wirklich eine gleiche Betheiligung beider Geschlechter an der Geburtenzahl in der Zeiteinheit vorhanden, so wäre gerade umgekehrt die nothwendige Folge dieser Erscheinung eine erhebliche Ungleichheit der Vertreter beider Geschlechter bei Zusammenfassung aller Altersstufen zu Gunsten des Ueberwiegens der Weiber. Für eln solches Ueberwiegen in der Zahl des weiblichen Geschlechtes fehlt es aber an jedem Grunde. Damit die Gleichheit in der Zahl der lebenden Individuen beider Geschlechter eintritt, deren Bestehen allein die Wahrscheinlichkeit verlangt, muss in der Zeiteinheit die Zahl der Geburten desjenigen Gèschlechtes überwiegen, welches zu seiner Ausreifung eine längere Zeit beansprucht. Die Grösse des Ueberwiegens der männlichen Geburten, welche wir, unter der Voraussetzung, dass es an jedem Grunde für eine Ungleichheit in der Zahl der Lebenden aller Altersclassen bei beiden Geschlechtern mangelt, nach dem Naturgesetze zu erwarten haben, wird aber eben durch die ,Sexualproportion" dargestellt. Es bestehen demnach zwei Ungleichheiten; eine Ungleichheit in der Reproductionsgeschwindigkeit zu Gunsten der Weiber und umgekehrt eine Ungleichheit in der Geburtenzahl zu Gunsten der Männer. Beide Ungleichheiten stehen zu einander in reciprokem Verhältnisse und heben sich gegenseitig auf mit der Wirkung der theoretisch verlangten Gleichheit der thatsächlich in einem grösseren Zeitabschnitt producirten Existenzen beider Greschlechter. Die eine Ungleichheit fällt sofort in die Augen und hat deshalb allein Beachtung gefunden, ohne dass man genügend berücksichtigt hat, dass sie nur die nothwendige Compensation für die andere mehr versteckte Ungleichheit entgegengesetzter Richtung ist.

Die vorliegende Darstellung wird aus zwei Gründen Befremden erwecken, zunächst wegen der herangezogenen Beweismethode und dann, weil der behandelte Gegenstand scheinbar eines jeden hygienischen Interesses ermangelt. Was den ersten Einwand betrifft, so muss zugegeben werden, dass auf naturwissenschaftlichem Forschungsgebiete für absehbare Zeiten der inductiv experimentellen Richtung unbedingt die Herrschaft 
gebührt. Aber in den biologischen Wissenschaften hat die inductive Methode ihre ausschliessliche Vorherrschaft nicht allein ihrer Erfolge wegen errungen, sondern zum grossen Theil deshalb, weil die Probleme einer mathematischen Behandlung selten einmal zugänglich sind. Jedoch selbst für die inductive Methode gilt der Satz, dass Observationes et numerandae sunt et perpendendae. Ohne deductive Kritik der erhaltenen Ergebnisse ist ein Fortschritt auch hier nicht möglich. Die vorliegende Frage ist aber eine solche, bei welcher ausnahmsweise eine deductive Behandlung möglich und bei welcher die Lösung einer scheinbar unerklärlichen Beobachtung einfach auf dem Wege der mathematischen Discussion erbracht werden konnte. Wäre es angängig gewesen, sich ausschliesslich der mathematischen Zeichensprache, statt des Hinweises auf gezeichnete Schemata zu bedienen, so wäre allerdings die Darstellung wesentlich einfacher geworden.

Dann aber entbehrt der Gegenstand durchaus nicht des hygienischen Interesses und zwar in zweifacher Hinsicht, wenn immerhin die Verbindungen nur mittelbare sind.

Schon in der Einleitung wurde betont, dass die Sterblichkeit der Kinder, namentlich an Infectionskrankheiten, zuweilen erhebliche Unterschiede nach den Geschlechtern aufweist. Begnügt man sich bei der Prüfung der Frage mit einem kleineren Materiale und beschränkt sich auf einzelne Krankheiten, so ergeben sich je nach den vergliehenen Lebensaltern so sehr widersprechende Resultate, dass man sich den Worten von Flügge ${ }^{1}$ anschliessen muss, nach welchen die Differenzen zwischen beiden Geschlechtern ,äusserst gering und schon deshalb einer sicheren Deutung kaum zugänglich sind." Bei der Betrachtung eines grösseren Materiales, z. B. der Kindersterblichkeit des ganzen preussischen Staates, dagegen wird der Unterschied des Antheils, welchen beide Geschlechter im kindlichen Alter an der Sterblichkeit haben, doch ein recht erheblicher, so dass er nicht vernachlässigt werden kann und man zur Voraussetzung verschiedener ursächlich einwirkender Momente kommen muss. Es sind solche Ursachen auch in der verschiedenen Art der Auferziehung, in der grösseren Lebensgefahr, welche dem wilderen Knaben drohe, u.s. w. gesucht worden, und es kann nicht bestritten werden, dass alle diese Punkte nicht gleichgültig sein werden. Aber die vorausgeschickte Betrachtung mahnt doch zur Vorsicht in Bezug auf die Tragweite der Schlussfolgerungen. Die Zahl der lebenden Mädchen einer Altersclasse kann nicht ohne Weiteres mit der der gleichalterigen Knaben in Vergleich gesetzt werden. Wenn die Mädchen, um wegen des Beispieles runde Zahlen zu

${ }^{1}$ C. Flügge, Die Verbreitungsweise der Diphtherie. Diese Zeitschr. Bd. XVII. 
wählen, mit 16, die Knaben etwa mit 20 Jahren den Endpunkt der Kindheit erreichen, so bedeutet ein Lebensjahr in dem Leben des Mädchens einen grösseren Bruchtheil der Kindheit als beim Knaben. Andererseits hat sich gezeigt, dass das weibliche Geschlecht sich schneller reproducirt als das männliche, dass demnach nicht die Zahl der Geburten, sondern das Product aus der ungleichen Geburtenzahl und dem ungleichen Reproductionsverhältniss erst einander gleich zu setzen sind. In einem grösseren Zeitabschnitt, etwa in einem Jahrhundert, wird eben die Zahl der Geborenen beider Geschlechter gleich; denn aus den zu Beginn dieses Zeitraumes erfolgten Geburten sind etwas mehr Mädchen bis zum Ablauf der Periode entstanden als Knaben und zwar im umgekehrten Verhältniss zur Sexualproportion. Auf das Kindesalter wirkt nun diese Ungleichheit der Generationsdauer noch nicht. Hier sind die Individuen noch geschlechtslos und darum fällt hier die Ungleichheit der Sexualproportion noch voll in's Gewicht. Hièr stehen zwar noch 1000 Mädchengeburten 1063 gleichaltrigen Knabengeburten gegenüber. Wenn aber im Alter von 0 bis 1 Jahr ron je 1000 Kindern beider Geschlechter je 100 Knaben und 100 Mädchen sterben, so ist der Verlust an Mädchen erheblicher, denn von 100 Mädchen entstehen im Laufe weniger Generationen mehr neue Individuen, als in dem gleichen Zeitraum von 100 Knaben und zwar im Verhältnisse der Sexualproportion.

Wenn also im preussischen Staate von Kindern im Alter von 1 bis 10 Jahren in den Jahren 1875 bis 1884 im 10jährigen Durchschnitt

$$
\begin{array}{lll}
\text { Knaben } & 35.6 & \text { Procent, } \\
\text { Mädchen } & 32.8 \quad "
\end{array}
$$

bis zur Erreichung des 10. Lebensjahres sterben, so erseheint der Unterschied sehr beträchtlich. Reducirt man ihn aber auf die Sexualproportion, so stehen den 32.8 Proc. der Mädchensterblichkeit nur noch 33.5 Proc. Knabensterblichkeit gegenüber; die Zurückführung der so viel geringeren Differenz auf zufällige Ereignisse, wie abweichende Erziehung und Lebensweise, wird jetzt viel einleuchtender.

Ein zweiter Gesichtspunkt ist noch weittragender. Das Problem der Sexualproportion wurde erst dadurch lösbar, dass das Individuum nicht als ein Wesen für sich, sondern als ein Product seiner Vorfahren und als ein Ausgangspunkt für neue Generationen aufgefasst wurde. Dieser Gesichtspunkt, das Einzelindividuum nur als einen Durchgangspunkt in der Entwickelung der Geschlechter und als einen Theil eines grossen Ganzen zu betrachten, ist so sehr der herrschende Punkt in den biologischen Wissenszweigen unseres Jahrhunderts geworden, dass es fast trivial erscheinen könnte, ihn noch besonders zu betonen. Wir haben ja 
alle diese Grundanschauung als Studenten schon nicht bloss in den Hörsälen, sondern selbst bei Commersen, z. B. in Scheffel's Gedicht vom Basalte, in uns aufgenommen. Merkwürdiger Weise aber hat die moderne Richtung der Hygiene, vor Allem auf dem Gebiete der Prophylaxe, sich von dieser Auffassung ferner gehalten, als für den Fortschritt wünschenswerth gewesen wäre. Der Entwickelungsgang dieser Wissenschaft, welche bisher fast ausschliesslich mit dem Ausbau der glänzenden Errungenschaften der bakteriologischen Methode beschäftigt war, macht diese Unterlassung begreiflich. Aber es konnte nicht ausbleiben, dass in Folge dessen der Gegensatz zwischen Individualhygiene und socialer, wie Rassenhygiene, immer auffälliger und dass eine Ausgleichung dieses Gegensatzes immer dringender erheischt wird. Das Verlangen Buchner's nach positiver Hygiene ist nichts Anderes, als ein Hinweis auf diese bisher übliche Unterlassung und namentlich A. Plötz hat in seinem jüngsten Werk: „Ueber Rassenhygiene“ systematisch an der Ausgleichung des Conflicts zwischen Individualhygiene und Rassenhygiene gearbeitet.

Der Weg, der für die Aufklärung der Sexualproportion zum Ziele fübrte, nämlich das Individuum nicht für sich zu betrachten, sondern als einen Durchgangspunkt in der Entwickelung der Generationen, verspricht auch auf dem Gebiete der Seuchenforschung reichliche Ergebnisse. Vor Allem sind es aber hier zwei Probleme, welche überhaupt nur auf diesem Wege dereinst zu lösen sein werden, nämlich die Frage nach der angeborenen Immunität und die Frage nach der Periodicität der Seuchen. 